\title{
Examination on the necessity of pericardial fat tissue resection in extended thymectomy for myasthenia gravis
}

\author{
Katsuhiro Okuda ${ }^{1}$, Hideo Hattori ${ }^{2}$, Keisuke Yokota ${ }^{1}$, Tsutomu Tatematsu ${ }^{1}$, Tadashi Sakane ${ }^{1}$, Risa Oda $^{1}$, \\ Takuya Matsui ${ }^{1}$, Ryoichi Nakanishi ${ }^{1}$ \\ ${ }^{1}$ Department of Oncology, Immunology and Surgery, Nagoya City University Graduate School of Medical Sciences, Nagoya, Japan; ${ }^{2}$ Department of \\ Pathology and Molecular Diagnostics, Nagoya City University Graduate School of Medical Sciences, Nagoya, Japan \\ Contributions: (I) Conception and design: K Okuda; (II) Administrative support: R Nakanishi; (III) Provision of study materials or patients: K Okuda, \\ H Hattori, K Yokota; (IV) Collection and assembly of data: T Tatematsu, T Sakane, R Oda, T Matsui; (V) Data analysis and interpretation: K Okuda, \\ H Hattori; (VI) Manuscript writing: All authors; (VII) Final approval of manuscript: All authors. \\ Correspondence to: Katsuhiro Okuda, MD, PhD. Nagoya City University Graduate School of Medical Sciences, 1 Kawasumi, Mizuho-cho, Mizuho- \\ ku, Nagoya 467-8601, Japan. Email: kokuda@med.nagoya-cu.ac.jp.
}

Background Extended thymectomy with pericardial fat tissue resection has been a mainstay in the treatment for myasthenia gravies (MG), but few studies have examined the necessity of the pericardial fat tissue resection in extended thymectomy.

Methods: We pathologically examined the distribution of germinal centers in the resected thymus including the thymus-surrounding fat tissue. Patients who underwent extended thymectomy using subxiphoid thoracoscopy for generalized MG or thymoma with anti-acetylcholine receptor antibody (antiAchR Ab) positivity from March 2015 to January 2021 were included in this study.

Results: A total of 20 patients underwent extended thymectomy (generalized nonthymomatous MG, $\mathrm{n}=5$; generalized MG with thymoma, $n=6$; thymoma with anti-AchR Ab positivity, $n=9)$. The resected specimens were divided to 6 parts and were pathologically examined to investigate the distribution of the geminal centers in all lesions. The number and distribution of germinal centers in the thymus varied depending on the patient, and no germinal centers were identified in the right or left pericardial fat tissues.

Conclusions: It is necessary to resect the pericardial fat tissue in patients whose preoperative images show the presence of pericardial fat tissue hyperplasia or ectopic thymoma. However, extensive pericardial fat tissue resection might not be necessary for all MG patients who undergo extended thymectomy with opening of the bilateral pleural by video-assisted or robot-assisted thoracoscopic surgery (RATS) via a subxiphoid approach.

Keywords: Myasthenia gravis (MG); extended thymectomy; pericardial fat tissue; germinal center; video-assisted thoracoscopic surgery (VATS)

Submitted May 12, 2021. Accepted for publication Jun 25, 2021.

doi: $10.21037 / g s-21-318$

View this article at: https://dx.doi.org/10.21037/gs-21-318

\section{Introduction}

The efficacy of thymectomy for myasthenia gravis (MG) with median sternotomy was reported by Blalock et al. in 1939 (1). Thymectomy was necessary to increase the rate of remission of MG; however, the optimal range of resection was ambiguous until Masaoka et al. (2) reported a high incidence of extracapsular thymic tissue in mediastinal fat and the need for extended resection with the thymus and all mediastinal fat. A few reports have compared the rate of remission of MG symptoms between patients received extended thymectomy and those who received limited resection and concluded the effectiveness of extended thymectomy $(3,4)$. Masaoka et al. reported that the rage 
of resection in extended thymectomy should include the pericardial fat tissue (3).

The gold standard surgical approach for extended thymectomy was thoracotomy by sternotomy, and a transcervical approach, video-assisted thoracoscopic surgery (VATS) via a lateral approach, and a subxiphoid approach have been applied (5-8). The therapeutic value of extended thymectomy via median sternotomy in nonthymomatous MG was proven by the Myasthenic Gravis Thymectomy Trial (MGTX) (9). At present, sternotomy is the only surgical approach in extended thymectomy for MG that has proven to be effective. However, it has been reported that VATS and robot-assisted thoracoscopic surgery (RATS) for anterior mediastinal tumors, including extended thymectomy for MG, are useful and are associated with fewer complications in comparison to thoracotomy due to the less invasive surgical approach (5-8).

Recently, preoperative imaging technology has been improving, and computed tomography (CT) with a $1-\mathrm{mm}$ slice thickness and sagittal or coronal reconstruction can be performed for patients undergoing surgery. Preoperative confirmation of abnormal findings around the thymus, including ectopic thymoma, has become easy to detect.

The amount of pericardial fat tissue differs in each patient, and the resection of the pericardial fat tissue is sometimes difficult because of the difficulty in securing the surgical field of view and surgical procedure. The surgical field through a thoracoscope in VATS improves visibility and the pericardial fat tissue extending to the diaphragm can be more clearly identified in comparison to thoracotomy.

There is a question as to whether it is necessary to remove all of the pericardial fat tissue that clearly shows the absence of thymic tissue on preoperative imaging and thoracoscopy. In order to avoid unnecessary organ damage, especially diaphragmatic nerve damage and unnecessary bleeding, we examined the distribution of geminal centers in the resected tissue from patients who had undergone extended thymectomy. Ambrogi et al. and Klimek-Piotrowska et al. reported that "active ectopic thymic tissue" was defined by the presence of germinal centers in ectopic thymic tissue $(10,11)$. We investigated the necessity of the pericardial fat tissue resection for all MG patients who underwent extended thymectomy in an era of advances in preoperative diagnostic imaging and an improved surgical field of view through thoracoscopic surgery. We present the following article in accordance with the STROBE reporting checklist (available at https:// dx.doi.org/10.21037/gs-21-318).

\section{Methods}

\section{Patients}

We investigated 20 patients of $<60$ years of age who underwent extended thymectomy for generalized MG or thymoma with anti-AchR Ab positivity at Nagoya City University Hospital from March 2015 to January 2021. We pathologically examined the distribution of germinal centers in the resected thymus and pericardial fat tissue. The study was conducted in accordance with the Declaration of Helsinki (as revised in 2013). The study was approved by the Institutional Review Board of Nagoya City University Hospital (IRB number: 70-19-0016) and each patient gave written consent for the use of their clinicopathological data for research.

\section{Pathological examination}

Figure $1 A-1 C$ shows a specimen resected by extended thymectomy. The pathologist (H.H.) evaluated the number of germinal centers in the all area of the resected thymus and pericardial fat tissue by hematoxylin and eosin staining and mainly investigated the presence or absence of germinal centers in the pericardial fat tissue.

\section{Patient follow-up}

The patients who underwent extended thymectomy were followed as outpatients after surgery. In 11 patients with generalized MG, with or without thymoma, we investigated the rate of remission of MG symptoms. In the other 9 patients without MG symptoms, we investigated whether the onset of MG occurred after the operation or not. In all patients, postoperative chest $\mathrm{CT}$ was checked within at least one year after surgery.

\section{Operation (surgical approach)}

The operation was performed by VATS or RATS via a subxiphoid approach (12). The thoracoscopic approach was performed using a $3-5 \mathrm{~cm}$ subxiphoid incision with one 5 -mm trocar inserted on the anterior axillary line in the right and left anterior fifth intercostal space. The robotassisted approach was performed in almost same incisions as the thoracoscopic approach, using a $3-5 \mathrm{~cm}$ subxiphoid incision with two 8-mm trocars at the right fifth intercostal space and one $8-\mathrm{mm}$ trocar at the left fifth intercostal space. At the end of the operation, it was confirmed by 


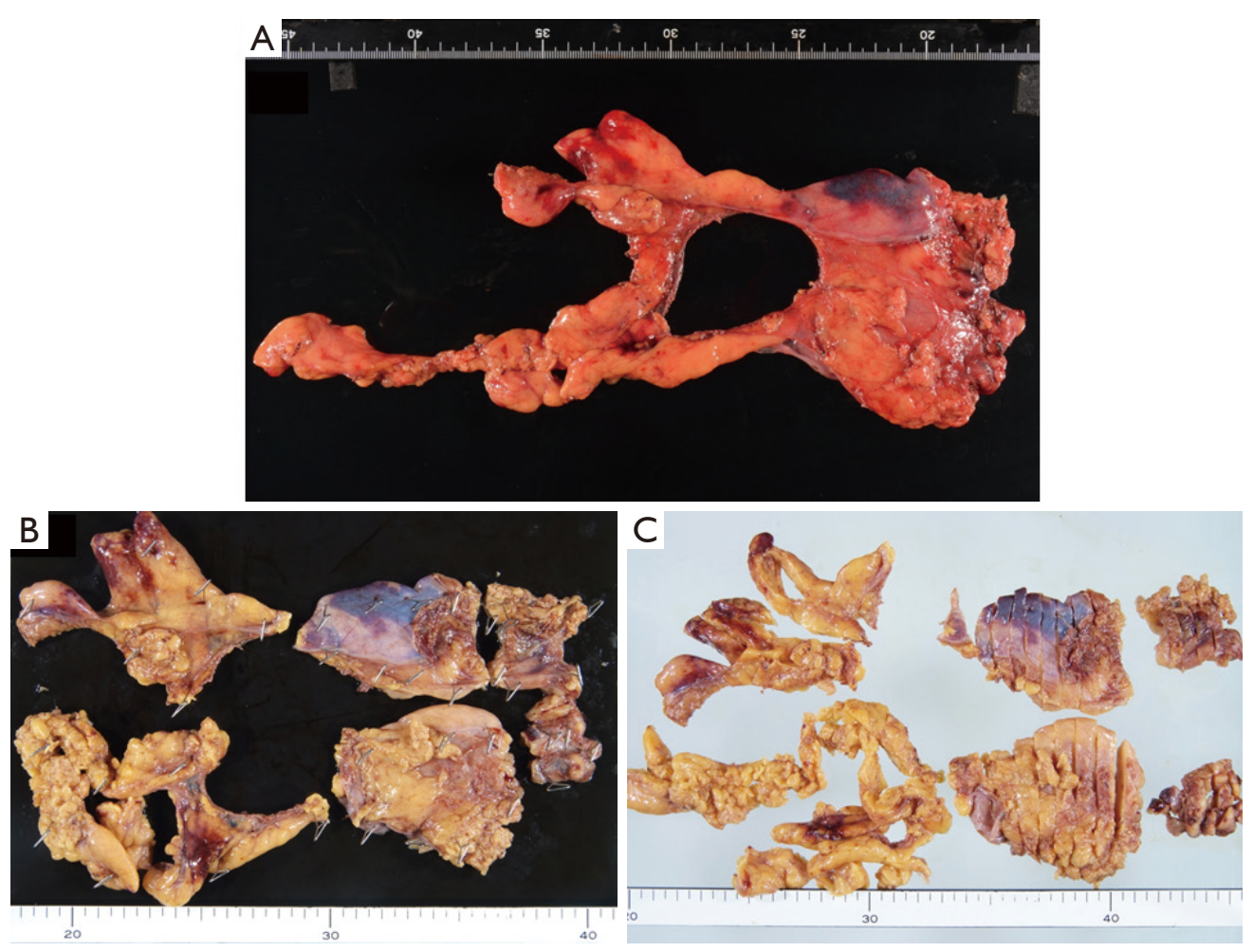

Figure 1 Thymic tissue (case 3) resected by extended thymectomy (A). The thymic tissue was divided into 6 parts (upper right lobe, upper left lobe, lower right lobe, lower left lobe, right pericardial fat tissue and left pericardial fa tissue) and embedded in paraffin blocks after fixed in $10 \%$ formalin (B). Tissue blocks were sliced (C) and stained using hematoxylin and eosin.

thoracoscope from the left and right ports that there was no residual pericardial fat tissue around the pericardium.

\section{Statistical analysis}

Fisher's test was performed for the presence of absence of germinal center and each factor. All statistical analyses were performed using the EZR software program (13), Significance was defined as a probability value of less than 0.05 .

\section{Results}

A total of 20 patients (generalized nonthymomatous MG, $\mathrm{n}=5$; generalized $M G$ with thymoma, $\mathrm{n}=6$; and thymoma with positive for anti-AchR Ab positivity, $n=9$ ) underwent extended thymectomy. Table 1 shows the characteristics of the 20 patients, including the distribution of germinal centers in each patient. All patients underwent extended thymectomy with thoracoscopy via a subxiphoid approach, including VATS $(n=14)$ and RATS $(n=6)$. All patients were discharged without perioperative complications.

Among the generalized MG patients, 6 patients had germinal centers in any resected tissue. In the generalized MG with thymoma patients, 2 of 5 patients had germinal centers in any resected tissue. In the patients with thymoma who were positive for anti-AchR Ab, 8 of 9 patients had germinal centers in any resected tissue.

Follow-up CT was performed within 1 year after the operation in all patients. No patients showed a residual thymic tumor or hyperplasia within the imaging range of chest CT. No obvious pericardial fat tissue could be confirmed on image. The median follow-up period was 32 months (range, 3-75 months). Although the distribution of the germinal centers depended on each patient, no germinal centers were found in the pericardial fat tissue.

After surgery, all patients with serum anti-AchR Ab showed a decreased antibody titer in comparison to before surgery. The remission rate of the 11 generalized MG patients was $45 \%$. Remission after surgery was achieved in all patients with generalized MG; 5 of the 11 patients showed complete remission (no need for medication), while 
Table 1 The list of 20 patients' characteristics including distribution of germinal center

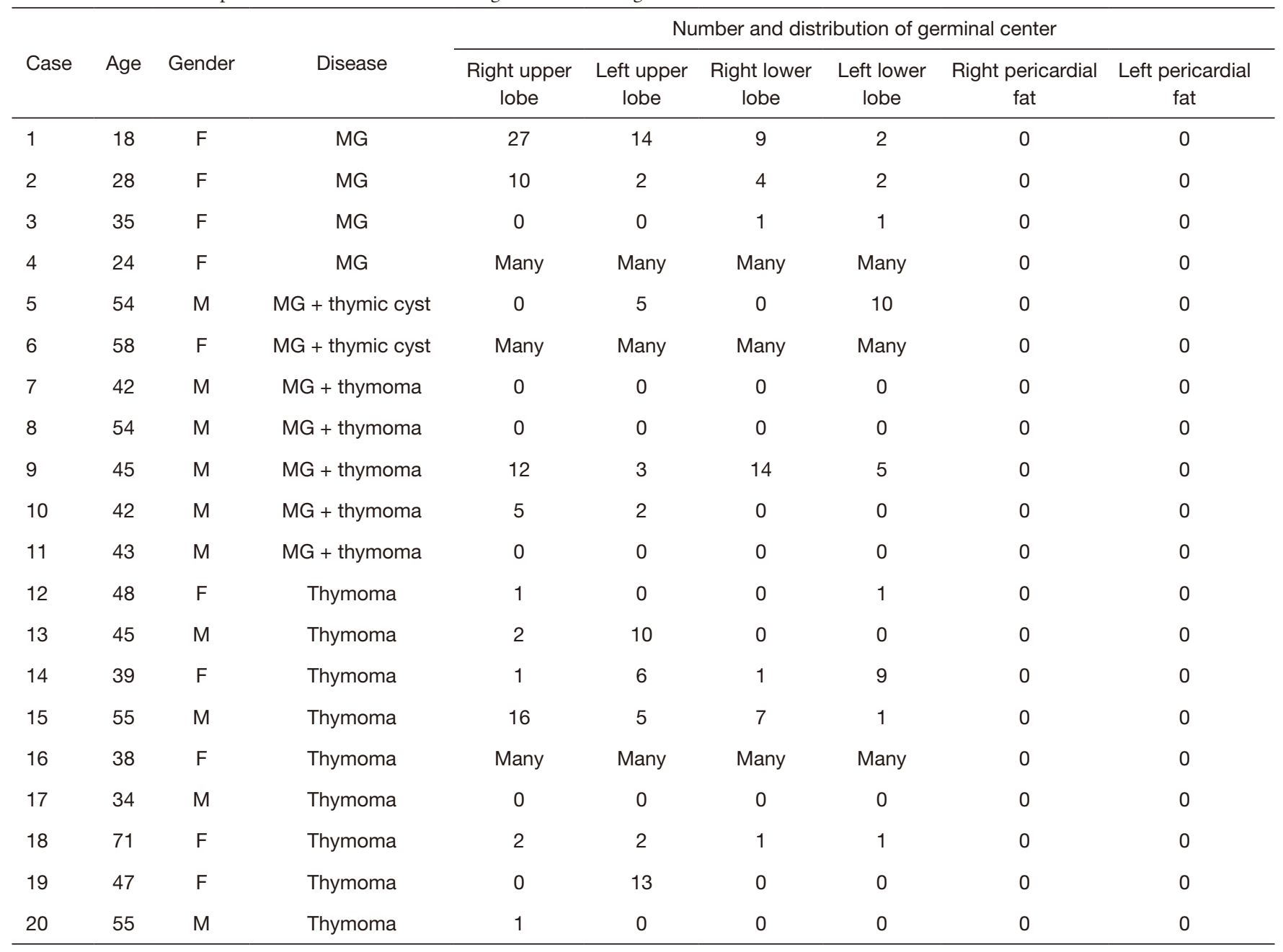

M, male; F, female, MG, myasthenia gravis.

the dosage of steroids or immunosuppressant agents could be reduced in the other 6 patients.

\section{Discussion}

It is widely known that ectopic thymus and active ectopic thymic tissue are present in the peri thymic fat tissue, and it has been pointed out that they interfere with the remission of MG (14-16). Some reports have examined the distribution of ectopic thymus in the peri-thymic fat tissue (17-22); however, few reports have examined the number or distribution of germinal centers in the peri thymic fat tissue $(10,11)$.

Because the field of view around the thymus is quite different depending on the surgical approach, for example thoracotomy $v s$. thoracoscopy or lateral approach $v s$. mediastinal $v s$. subxiphoid approach, the range of excision must be decided according to the surgical technique and approach. VATS and RATS using the subxiphoid approach provide a better view around the neck, both sides of the phrenic nerves, and the pericardium in comparison to thoracotomy and lateral thoracoscopy, because the view from both thoracic cavities can be seen. In some cases, the fat tissue around the thymus is quite abundant, and it is difficult to determine how much fat tissue should be removed. Pericardial fat tissue with no abnormal findings on preoperative imaging is just fat tissue without thymic tissue; thus, it would not be expected to affect MG. We wondered whether such tissue should be removed with extended resection of the pericardial fat tissue or not because unnecessary bleeding and organ injury, including 
injury of both the right and left phrenic nerves, should be avoided. The present study was undertaken to solve the above question. The surgical procedure was changed from thoracotomy to thoracoscopic surgery, so that the range of resection surrounding the thymus suitable for thoracoscopic surgery should be confirmed.

In this study, we investigated the distribution of the germinal centers in the thymus after resection by extended thymectomy and considered the region of pericardial fat tissue resected in extended thymectomy, which was performed via a thoracoscopic approach for the treatments of MG. The tissues removed by extended thymectomy were divided into 6 parts, and a pathological examination was performed to detect germinal centers in each part. The number and distribution of germinal centers in the thymus differed in each case. On the other hand, we did not find ectopic thymus or germinal centers in the pericardial fat tissue of any of the 20 patients. Considering the improvement in the accuracy of preoperative imaging and the improvement in the surgical view the use of a thoracoscope, it has become easier to reliably determine the dissection line around the cardio-phrenic angle. In subxiphoid thoracoscopic resection, the range of resection, which includes both pleura is extended in conventional thoracotomy. It is necessary to re-examine the range of excision by VATS and RATS in comparison to conventional thoracotomy.

We found that there were no germinal centers in the pericardial fat tissue of all 20 patients who received extended thymectomy by VATS or RATS via a subxiphoid approach. Proof that it is not necessary to remove all of the continuous pericardial fat tissue would enable safer surgical resection. It has been reported that surgical resection including the bottom of thymus is not sufficient to achieve complete remission in all MG patients (2,17-19,21-23); however, resection of the apparent fat tissue region of the pericardial fat tissue would be sufficient. Of course, surgeons check preoperative imaging, including CT and MRI in order to investigate the existence of ectopic thymoma or thymic hyperplasia around the pericardial fat tissue.

The preset study was associated with some limitations. First, the number of patients was relatively small. The enrolled patients were not limited to patients with nonthymomatous MG. Although it would be desirable to limit the number of patients with nonthymomatous MG, it was difficult to collect patients with nonthymomatous MG because of the rarity of the disease and the fact that the study was limited to a single-center. Second, due to the relatively small number of patients and the short postoperative observation period, postoperative MG symptoms could not be fully evaluated. Third, it was difficult to objectively evaluate the adequate range of resection of pericardial fat tissue. Finally, this study was retrospective study.

Although this study only included 20 cases, the findings may suggest that extensive pericardial fat tissue excision may not be necessary for all MG patients who undergo extended thymectomy with opening of the bilateral pleura by VATS or RATS via a subxiphoid approach.

\section{Conclusions}

In this study, we found no ectopic thymic tissue or germinal centers in the pericardial fat tissue. It may be possible to reduce the range of excision of pericardial fat tissue in patients treated by VATS or RATS via a subxiphoid approach. In the future, we should demonstrate the effectiveness of extended thymectomy by VATS or RATS for MG patients to investigate the appropriate extent of resection.

\section{Acknowledgments}

Funding: None.

\section{Footnote}

Reporting Checklist: The authors have completed the STROBE reporting checklist. Available at https://dx.doi. org/10.21037/gs-21-318

Data Sharing Statement: Available at https://dx.doi. org/10.21037/gs-21-318

Peer Review File: Available at https://dx.doi.org/10.21037/ gs-21-318

Conflicts of Interest: All authors have completed the ICMJE uniform disclosure form (available at https://dx.doi. org/10.21037/gs-21-318). The authors have no conflicts of interest to declare.

Ethical Statement: The authors are accountable for all aspects of the work in ensuring that questions related to the accuracy or integrity of any part of the work are appropriately investigated and resolved. The study was 
conducted in accordance with the Declaration of Helsinki (as revised in 2013). The study was approved by the Institutional Review Board of Nagoya City University Hospital (IRB number: 70-19-0016) and each patient gave written consent for the use of their clinicopathological data for research.

Open Access Statement: This is an Open Access article distributed in accordance with the Creative Commons Attribution-NonCommercial-NoDerivs 4.0 International License (CC BY-NC-ND 4.0), which permits the noncommercial replication and distribution of the article with the strict proviso that no changes or edits are made and the original work is properly cited (including links to both the formal publication through the relevant DOI and the license). See: https://creativecommons.org/licenses/by-nc-nd/4.0/.

\section{References}

1. Blalock A, Mason MF, Morgan HJ, et al. Myasthenia gravis and tumors of the thymic region: report of a case in which the tumor was removed. Ann Surg 1939;110:544-61.

2. Masaoka A, Nagaoka Y, Kotake Y. Distribution of thymic tissue at the anterior mediastinum. Current procedures in thymectomy. J Thorac Cardiovasc Surg 1975;70:747-54.

3. Masaoka A, Monden Y. Comparison of the results of transsternal simple, transcervical simple, and extended thymectomy. Ann N Y Acad Sci 1981;377:755-65.

4. Matell G, Lebram G, Osterman PO, et al. Follow up comparison of suprasternal vs. transsternal method for thymectomy in myasthenia gravis. Ann N Y Acad Sci 1981;377:844-5.

5. Sakamaki Y, Oda T, Kanazawa G, et al. Intermediate-term oncologic outcomes after video-assisted thoracoscopic thymectomy for early-stage thymoma. J Thorac Cardiovasc Surg 2014;148:1230-1237.e1.

6. Pompeo E, Dauri M, Massa R, et al. Minimalist thoracoscopic resection of thymoma associated with myasthenia gravis. J Thorac Cardiovasc Surg 2017;154:1463-5.

7. Shiomi K, Kitamura E, Ono M, et al. Feasible and promising modified trans-subxiphoid thoracoscopic extended thymectomy for patients with myasthenia gravis. J Thorac Dis 2018;10:1747-52.

8. Suda T, Hachimaru A, Tochii D, et al. Video-assisted thoracoscopic thymectomy versus subxiphoid single-port thymectomy: initial results $\uparrow$. Eur J Cardiothorac Surg 2016;49 Suppl 1:i54-8.
9. Wolfe GI, Kaminski HJ, Aban IB, et al. Randomized Trial of Thymectomy in Myasthenia Gravis. N Engl J Med 2016;375:511-22.

10. Ambrogi V, Mineo TC. Active ectopic thymus predicts poor outcome after thymectomy in class III myasthenia gravis. J Thorac Cardiovasc Surg 2012;143:601-6.

11. Klimek-Piotrowska W, Mizia E, Kuzdzał J, et al. Ectopic thymic tissue in the mediastinum: limitations for the operative treatment of myasthenia gravis. Eur J Cardiothorac Surg 2012;42:61-5.

12. Okuda K, Haneda H, Yokota K, et al. Thymothymectomy with pulmonary partial resection using the subxiphoid approach: how to do it? Surg Today 2018;48:1096-9.

13. Kanda Y. Investigation of the freely available easy-touse software 'EZR' for medical statistics. Bone Marrow Transplant 2013;48:452-8.

14. Papatestas AE, Genkins G, Kornfeld P. Comparison of the results of the transcervical and transsternal thymectomy in myasthenia gravis. Ann N Y Acad Sci 1981;377:766-78.

15. Fukai I, Funato Y, Mizuno T, et al. Distribution of thymic tissue in the mediastinal adipose tissue. J Thorac Cardiovasc Surg 1991;101:1099-102.

16. Ambrogi V, Tacconi F, Sellitri F, et al. Subxiphoid completion thymectomy for refractory non-thymomatous myasthenia gravis. J Thorac Dis 2020;12:2388-94.

17. Zieliński M, Kuzdzał J, Szlubowski A, et al. Transcervicalsubxiphoid-videothoracoscopic "maximal" thymectomy-operative technique and early results. Ann Thorac Surg 2004;78:404-9; discussion 409-10.

18. Zieliński M, Kuzdzal J, Szlubowski A, et al. Comparison of late results of basic transsternal and extended transsternal thymectomies in the treatment of myasthenia gravis. Ann Thorac Surg 2004;78:253-8.

19. Ponseti JM, Gamez J, Vilallonga R, et al. Influence of ectopic thymic tissue on clinical outcome following extended thymectomy in generalized seropositive nonthymomatous myasthenia gravis. Eur J Cardiothorac Surg 2008;34:1062-7.

20. Meacci E, Cesario A, Margaritora S, et al. Thymectomy in myasthenia gravis via original video-assisted inframammary cosmetic incision and median sternotomy: longterm results in 180 patients. Eur J Cardiothorac Surg 2009;35:1063-9; discussion 1069.

21. Pompeo E, Tacconi F, Massa R, et al. Long-term outcome of thoracoscopic extended thymectomy for nonthymomatous myasthenia gravis. Eur J Cardiothorac Surg 2009;36:164-9.

22. Tomulescu V, Sgarbura O, Stanescu C, et al. Ten-year 
results of thoracoscopic unilateral extended thymectomy performed in nonthymomatous myasthenia gravis. Ann Surg 2011;254:761-5; discussion 765-6.

Cite this article as: Okuda $\mathrm{K}$, Hattori H, Yokota K, Tatematsu T, Sakane T, Oda R, Matsui T, Nakanishi R. Examination on the necessity of pericardial fat tissue resection in extended thymectomy for myasthenia gravis. Gland Surg 2021;10(8):24382444. doi: $10.21037 /$ gs-21-318
23. Mineo TC, Pompeo E, Lerut TE, et al. Thoracoscopic thymectomy in autoimmune myasthesia: results of leftsided approach. Ann Thorac Surg 2000;69:1537-41. 